\title{
An Overview of the James Webb Space Telescope (JWST) Project
}

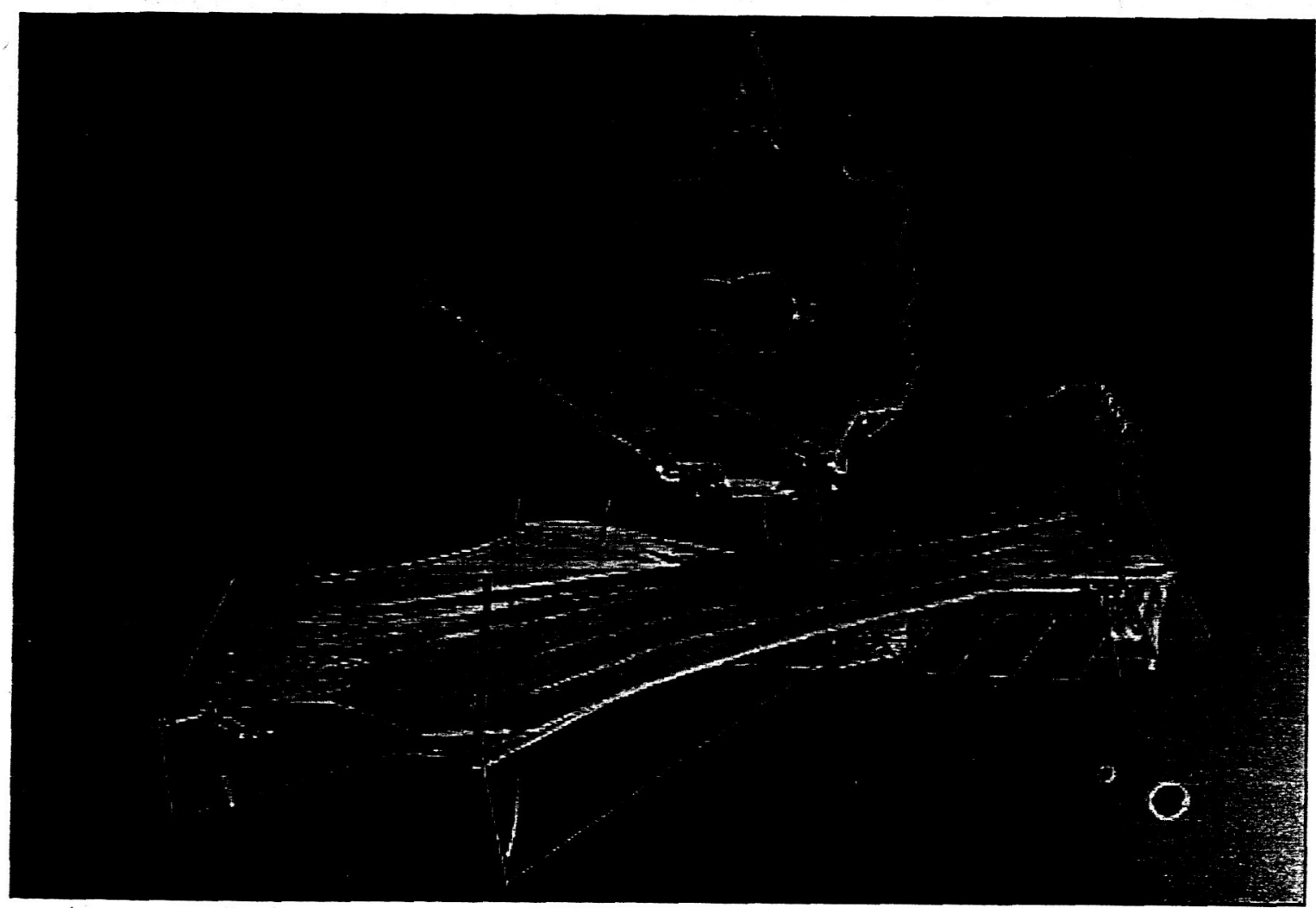

June 21-25, 2004

Phillip A. Sabelhaus, Project Manager, NASA Goddard Space Flight Center 


\section{ABSTRACT}

Authors:

Phillip A. Sabelhaus, JWST Project Manager 301-286-5712 phil.sabelhaus@nasa.gov

NASA, Goddard Space Flight Center, Code 443, Greenbelt, Md. 20771 Fax: 301-286-5558

John Decker, JWST Deputy Project Manager 301-286-5872 John.E.Decker@nasa.gov

NASA, Goddard Space Flight Center, Code 443, Greenbelt, Md. 20771 Fax: 301-286-5558

Presenter Bio:

\begin{tabular}{ll} 
Mr. Sabelhaus graduated from the University of Maryland with a \\
BS in Mechanical Engineering and came to work at the Goddard \\
Space Flight Center (GSFC) in 1978. In 1981, Mr. Sabelhaus \\
went to work as the Launch Vehicle Integration Manager for GTE \\
$\begin{array}{l}\text { Spacenet Corporation. He returned to GSFC in 1985 to work on } \\
\text { the NASA Space Station Program. In 1989, he joined the Flight } \\
\text { Programs and Projects Directorate (FPPD) at the GSFC, Code } \\
\text { 400. Since then, Mr Sabelhaus has served a Deputy Project } \\
\text { Manager, Project Manager and Program Manager in the FPPD } \\
\text { and is currently the Deputy Associate Director for the James } \\
\text { Webb Space Telescope (JWST). He led the teams that } \\
\text { successfully launched the TOM-EP, Landsat 7 and the EOS Aqua } \\
\text { satellites. He is married and has two daughters. }\end{array}$ \\
\hline
\end{tabular}

Keywords: James Webb; Space Telescope; JWST; Hubble; HST

The JWST project at the GSFC is responsible for the development, launch, operations and science data processing for the James Webb Space Telescope. The JWST project is currently in phase B with its launch scheduled for August 2011. The project is a partnership between NASA, ESA and CSA. The U.S. JWST team is now fully in place with the recent selection of Northrop Grumman Space Technology (NGST) as the prime contractor for the telescope and the Space Telescope Science Institute (STScI) as the mission operations and science data processing lead. This paper will provide an overview of the current JWST architecture and mission status including technology developments and risks.

Primary Mirror

\section{Mission in Brief}

6.5 Meter Class Segmented, Actively Controlled, $40^{\circ}$ Kelvin nominal temperature

Wavelength Range

0.6 to $28 \mu \mathrm{m}$

Instruments

Near-Infrared Multi-Object Spectrometer

Near-Infrared Camera

Near-Infrared Tunable Filter Camera

Mid-Infrared Camera/Spectrometer

Payload Mass

Aproximately $6,190 \mathrm{~kg}, 13,600 \mathrm{lbs}$. (Includes the payload adapter)

Launch Vehicle

Ariane V Expendable Launch Vehicle (TBR) 
Orbit

Lagrange Point 2 (1.5-million $\mathrm{km}$ or 940,000 miles from Earth)

Mission Length

5 years ( 10 year goal)

\section{INTRODUCTION}

The James Webb Space Telescope was conceived as a follow-on mission to the highly successful Hubble Space Telescope to allow scientists to see the first generations of stars.

Equipped with a large 18-segment, 6.5-meter primary mirror and a suite of revolutionary, infrared-sensing cameras and spectrometers, JWST will allow us to see younger objects in space than is currently possible with Hubble and help us analyze the miniscule specks of light that Hubble cannot detect. These nascent stars and galaxies are so distant that by the time their light reaches us, it has stretched into the longer, redder wavelength bands that are invisible to the human eye.

Consequently, no one has ever observed this cosmic "dark zone" before because they did not have the tools to do so. But with this "first light machine," we will finally see what the universe looked like when it was merely a fraction of its current age and size, when the first stars and galaxies were just beginning to take form and ignite. In addition to conducting this unprecedented science, JWST will demonstrate revolutionary new technologies needed for future Origins missions. For this reason, the National Academy of Science has ranked JWST as one of NASA's top science goals for this decade.

\section{ADDITIONAL SCIENTIFIC GOALS}

In addition to observing these young galaxies, JWST will tackle four other major objectives over the course of its 5-10 year lifetime. It will help determine the geometry of the universe, its age and determine its ultimate fate. A few years ago, two teams of astronomers rocked the scientific world by finding evidence that the expansion of the universe is accelerating rather than slowing down because of the gravitational attraction between the matter within it. Their observations seemed to confirm the existence of a new form of energy that causes the expansion of the universe to accelerate. JWST is capable of studying this phenomenon.

Although mission engineers designed the spacecraft to primarily peer at the farthest reaches of the universe, it will also look closer to home. With JWST, scientists can study the history of the Milky Way and its nearby neighbors by studying the old stars and star remnants that formed over the galaxy's lifetime. Astronomers will use JWST to study star birth and formation. Its infrared sensors can pierce the dust and gas that surround stellar nurseries and reveal the processes that dictate the mass and composition of stars, as well as the production of heavy elements. And last, NASA designed JWST to study the origin and evolution of planetary systems like our own. JWST may be able to directly detect large, Jupiter-sized planets around nearby stars. Although we cannot image smaller planets directly, JWST's high resolution will make it possible to see how planetary systems behave especially when they are in the process of formation, which will give us a more accurate picture of their evolution.

\section{JWST's Five Scientific Themes}

- Cosmology and the Structure of the Universe

- Origin and Evolution of Galaxies

- History of the Milky Way and its Neighbors 
- Birth and Formation of Stars

- Origin and Evolution of Planetary Systems

\section{Technological Challenges At-A-Glance}

1. A 6-meter-class, lightweight, cryogenic deployable, actively controlled with diffraction limited performance at a 2 micron wavelength.

2. A tennis court-sized deployable sunshield to passively cool the telescope and instruments to 40 degrees Kelvin providing sensitivity to extremely faint infrared photons.

3. A highly capable Observatory that weighs about $6,000 \mathrm{~kg}$, including the telescope and instruments.

4. Low-noise, large-area infrared detectors.

5. A cryogenic programmable slit mask for multi-object spectroscopy.

\section{ORBITAL CONSIDERATIONS}

JWST's orbit - at the second Lagrange Point (L2), located 1.5-million km (940,000 miles) from the Earth in the anti-Sun direction allows NASA to perform this mission. The L2 orbit offers a thermally stable environment. At the L2 point, JWST will be in orbit around the Sun rather than the Earth, as it is with HST. This arrangement will allow JWST to live in the shadow of a giant sunshield, which deploys on orbit. In this shadow, the JWST can passively cool the instruments to below $40^{\circ} \mathrm{Kelvin}$ (about $400^{\circ}$ below zero Fahrenheit) and the telescope optics to below $50^{\circ} \mathrm{Kelvin}$. Although passive cooling represents an old concept, NASA has never flown a mission before that uses this method to reach these extreme temperatures. (As a reference, WMAP (at L2) uses passive cooling to reach about $95^{\circ} \mathrm{Kelvin}$ )

\section{Lagrange Point (L2)}

\begin{tabular}{|l|l|}
\hline $\begin{array}{l}\text { The Sun, Earth and Moon will always be } \\
\text { on the same side of the telescope making it } \\
\text { easier for the sunshield to keep light and } \\
\text { heat away from the telescope cooling it to } \\
\text { the very low temperatures required to } \\
\text { prevent the telescope's own heat radiation } \\
\text { from exceeding the brightness of the } \\
\text { distant astronomical objects. }\end{array}$ \\
\hline
\end{tabular}




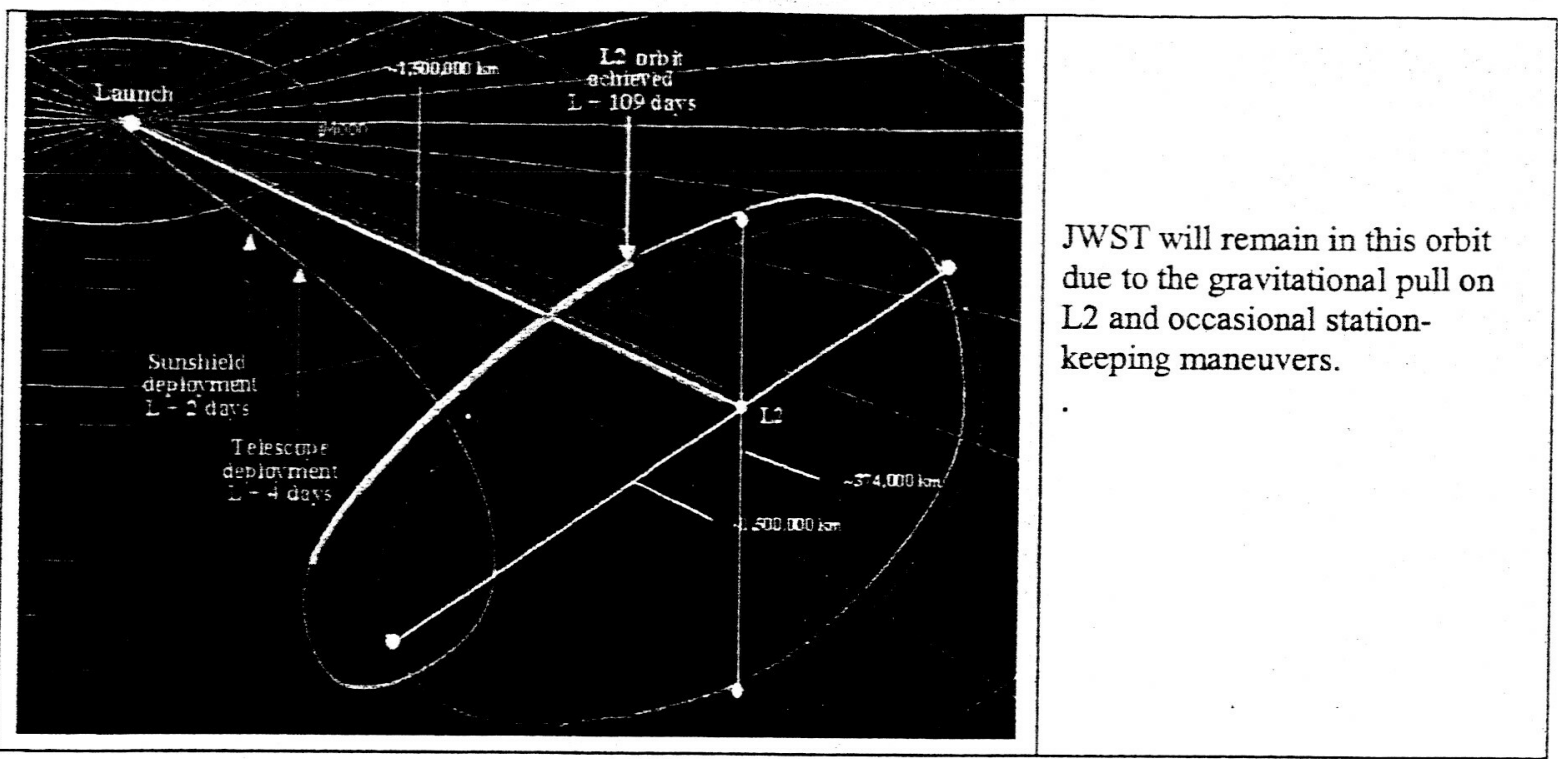

\section{CRYOGENIC COOLING}

To observe the farthest reaches of the universe, temperature is an essential consideration. We cannot observe in the - mid-infrared wavelength bands (0.6-0.9 microns is the visible to 28 microns) with the telescope at temperatures above $40^{\circ} \mathrm{Kelvin}$. At temperatures warmer than this the observatory would create too much of its own infrared "noise" or heat and interfere with JWST's attempt to detect extremely weak infrared photons.

JWST will passively cool to its cryogenic operating temperature by using a system of radiators shaded by the highly efficient tennis court sized sunshield. Early concept studies clearly indicated that actively cooling the telescope and instruments with mechanical coolers or stored cryogens would lead to mass and volume requirements incompatible with all launch vehicles. Separating and thermally isolating the warm spacecraft components to the sun side of the shield via a deployable isolation tower further enhance passive cooling. Power dissipation estimates on the cryogenic side of the observatory are judiciously monitored and allocations are stringently held to less than 300 milli-Watts. Although passively cooled to a quite cold temperature of 37 Kelvin, the mid-Infrared instrument will utilize a dewar of solid Hydrogen to further cool its detectors to less than 7 Kelvin.

\section{SUNSHIELD TECHNOLOGY}

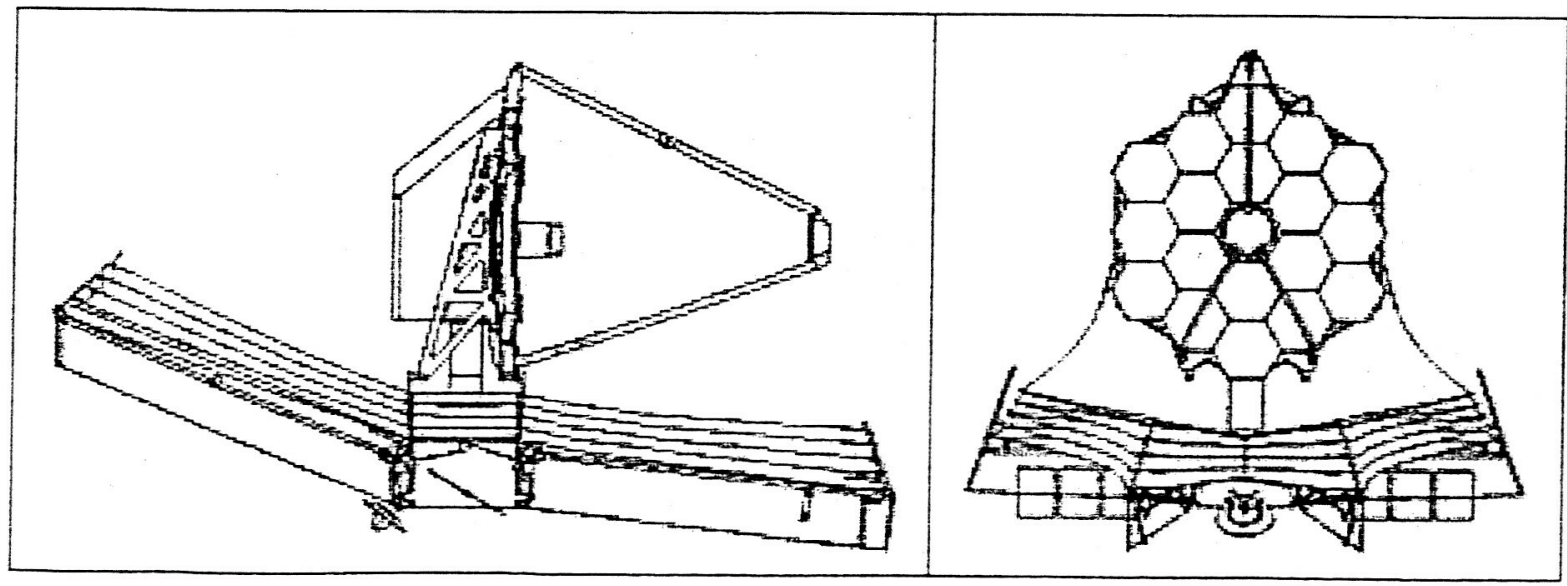


The sunshield assures the infrared telescope and sensors are fully protected from solar exposure and radiant heat sources generated by the spacecraft bus and solar arrays. The sunshield design concept consists of 5 separated layers of Kapton $E$ with silicon and vapor deposited aluminum (VDA) coatings. A highreliability deployment system consisting of eight articulating motor driven booms deploys and separates the layers. The shape and size of the shield is driven by the need to provide a large field of regard (FOR) while minimizing solar pressure momentum gain.

\section{COLD MIRRORS AND MOTORS}

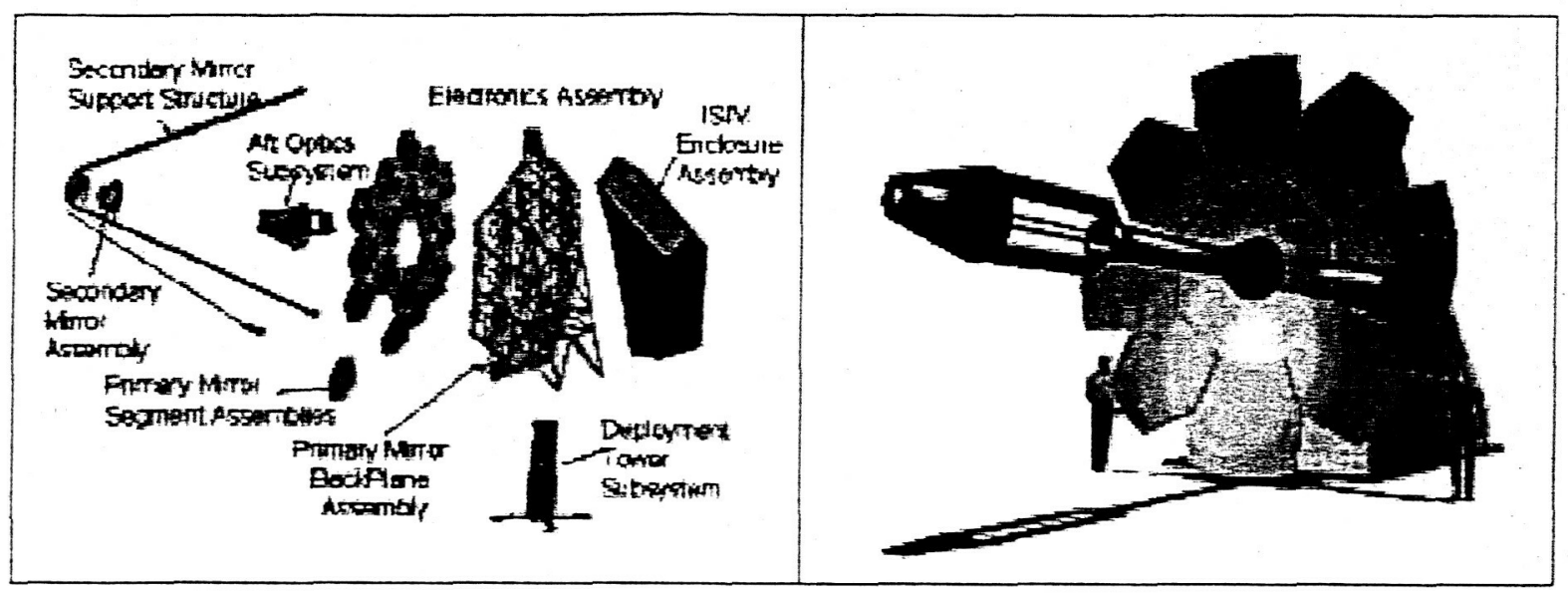

One of the challenges for the JWST team is to design and manufacture lightweight mirrors and an active mirror control system to enable the best possible scientific return by providing diffraction limited performance for all wavelengths greater than 2 microns.

Even more technologically demanding than the deployable sunshield is JWST's segmented, 6.5-meter lightweight, deployable mirror, which will do what no other spaceborne mirror has done - it will use a combination of small, ultra-precise actuators and sophisticated computer algorithms to align and properly figure the mirror. Developing such a technology is no small task - especially considering the added challenge of making JWST's primary mirror with six times the collecting area of Hubble's with more than a factor of 9 lower areal density $\left(\mathrm{kg} / \mathrm{m}^{2}\right)$. In contrast, Hubble's 2.4-meter primary mirror is a single piece of polished glass weighing $180 \mathrm{~kg}$ per square meter. Although the segmented approach using lightweight materials accomplishes the performance objectives and allows the telescope to fit inside a commercial rocket fairing, it complicates the task of making sure the mirror achieves and holds its proper shape.

JWST's architecture requires that the optical system wavefront errors be measured and then corrected while the spacecraft is on orbit. A perfect mirror is not required at launch, and any changes due to stress as a result of rocket vibrations, or the deployment and the cool-down to the extremely cold operating temperature can be corrected. The Hubble's well-known spherical aberration has given the scientific community a great deal of experience in determining an optical system's wavefront error using images in a process called phase diverse phase retrieval. Image-based phase retrieval is the technique of choice for JWST, and is the front half of the full wavefront sensing and control process.

Once in orbit, JWST will take several images of stars as part of wavefront error sensing that begins the process of aligning the mirrors. With those images, ground controllers will use sophisticated computer algorithms to determine the level of distortion in the mirror segments caused by the super-cold temperatures, misalignments and fabrication errors. Ground controllers will correct the calculated distortion by activating computer-controlled mechanical actuators that move and deform the mirror segments until they are perfectly aligned and shaped. Their goal is to reduce the RMS magnitude of these distortions to no 
more than 0.15 microns, 300 times smaller than the width of a human hair (for reference, HST's distortionThe outer edge of the mirror was ground too flat by a depth of 4 microns (roughly equal to one fiftieth the thickness of a human hair). These actuators need to work in extremely cold temperatures, which adds another level of diligence to the engineering task. This on-orbit wavefront sensing and control now under development at NASA and the prime contractor will undoubtedly find applications in other NASA and Defense Department missions.

Northrop Grumman has chosen the beryllium-based mirror technology made by Ball Aerospace \& Technologies Corporation as the primary mirror material for JWST.
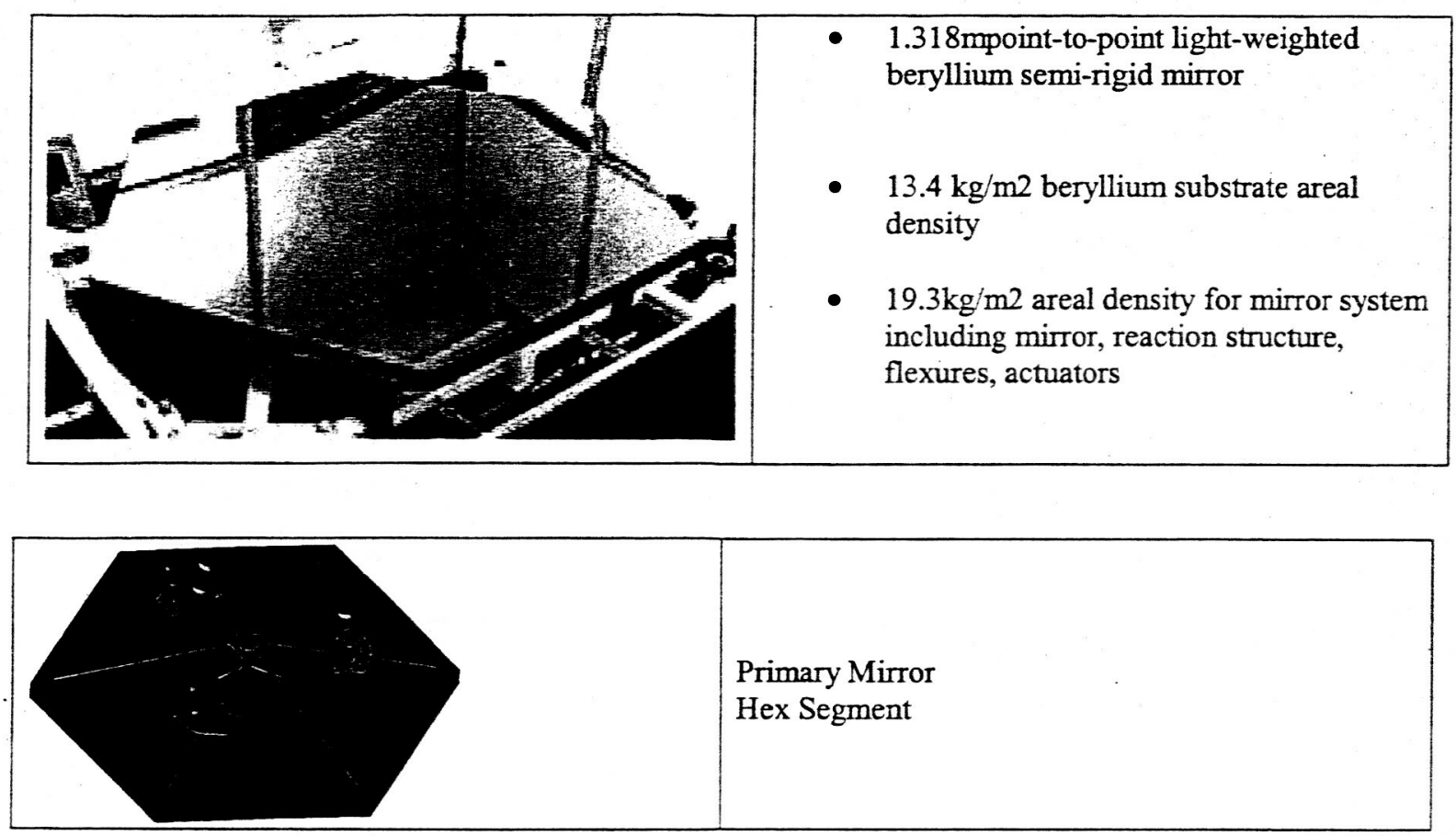

Ball Aerospace of Boulder, Colorado will develop the semi-rigid primary mirror design they pioneered in NASA's Advanced Mirror System Development (AMSD) program. With its seven actuators, this mirror design has enough degrees of freedom to provide on-orbit correction. In addition to AMSD, a beryllium primary mirror is in use on the Spitzer Space Telescope ((formerly known as the Space Infrared Telescope Facility (SIRTF)), providing NASA flight experience with meter-sized beryllium mirrors.

\section{INTEGRATED SCIENCE INSTRUMENT MODULE}

The telescope will carry four instruments and an ultra-precise fine guidance sensor - the Near Infrared Camera (NIRCam), the Near Infrared Spectrometer (NIRSpec), the Tunable Filter near infrared camera, the Mid Infrared Instrument (MIRI), and the Fine Guidance Sensor. The Near Infrared Camera (NIRCam) provided by a team led by the University of Arizona, will be JWST's primary imager in the wavelength range of 0.6 to 5 microns. Required by many of the core science goals, the instrument is particularly well suited for detecting the first light-emitting objects that formed after the Big Bang. It also will come equipped with a coronagraph, which scientists will use to obtain images of debris disks, like our own Kuiper Belt, and massive giant planets around nearby stars.

The multi-object Near Infrared Spectrometer (NIRSpec), provided by the European Space Agency, will serve as the principal spectrograph in the $0.6-$ to 5 -micron wavelength range. Spectroscopy is the study of light after it has been separated into its component colors revealing the composition, temperature and other 
physical characteristics of celestial objects. Its ability to obtain simultaneous spectra of more than 100 objects in a 9-square arc-minute field of view particularly interests the scientific community. By obtaining data on many objects in one observation, astronomers can better characterize the early universe and increase their chances to find rare and unique objects through a factor of 1000 increase in observing efficiency.

The Tunable Filter camera, provided by the Canadian Space Agency, will enable astronomers to program the filter of the camera to any narrow color range which will allow them to isolate objects with special color features, particularly in the very distant universe.

The Mid Infrared Instrument, provided by an international collaboration led by teams from the Jet Propulsion Laboratory and Europe, will provide imaging and spectroscopy at wavelengths of 5 to 28 microns. This capability opens a new window of discovery for scientists. This instrument will study the creation of the first heavy elements and the formation and evolution of galaxies and very old stellar populations. It is uniquely capable of studying the very early stages of star and planet formation, in regions where all visible light is blocked by dust and most of the emission is radiated at mid-infrared wavelengths.

The Fine Guidance Sensor, also provided by the Canadian Space Agency, will ensure the telescope can precisely point to a few milli-arcseconds of angle and find even faint guide stars in the darkest regions of space for at least $95 \%$ of all desirable scientific observations.

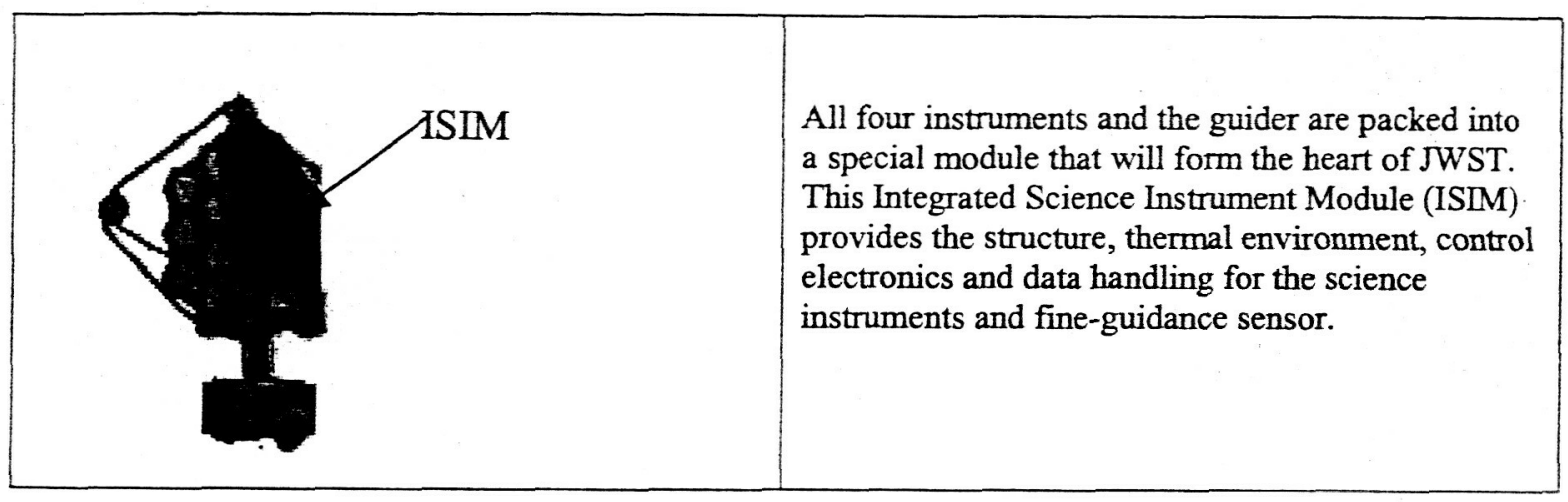

JWST's ISIM features two groundbreaking technologies - iarge-format detectors for all three instruments and a programmable spectrometer aperture mask (micro shutters) for the Near-Infrared Spectrometer. They are vital for carrying out the projects rigorous scientific program

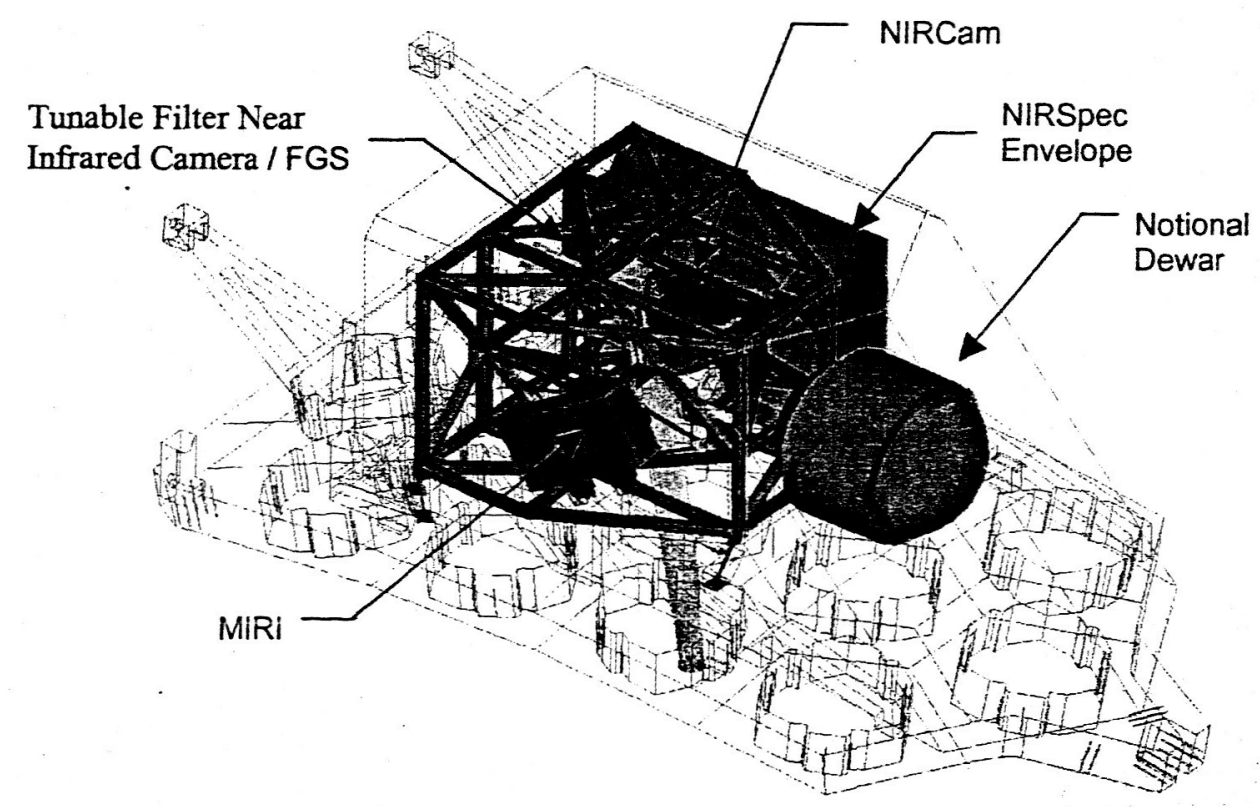




\section{DETECTORS}

More than any other component, the detector determines the sensitivity of an instrument. Its role is to record the position, intensity and, by means of filters and spectrographs, the wavelength of as much of the incident radiation as possible. Because JWST's prime targets are intrinsically faint, with fluxes as low as a single photon arriving every second, its detectors must be more sensitive than any detector ever flown. Furthermore, because the detectable first star-forming regions in the universe are very rare, JWST must be able to image large areas of the sky and JWST's detector assemblies must be large mosaic arrangements of up to 16 million pixel per focal plane array (FPA) for a total of 67 mega pixels.

JWST's near-infrared instruments have selected Rockwell HAWAII-2RG (H2RG) mercury-cadmiumtelluride ( $\mathrm{HgCdTe}$ ) detectors. These detectors, which represent the state-of-the-art in this wavelength regime, are the fruit of a highly successful JWST's Phase-A technology development program. Under this program, Rockwell and Raytheon developed $2 \mathrm{~K} \times 2 \mathrm{~K}$ sensor chip assemblies (SCAs) and prototype FPAs. JWST candidate SCAs were tested at the vendors affiliated labs at the University of Hawaii and University of Rochester respectively, and independently at the Space Telescope Science Institute. Upon completion of the technology development program, NIRCam selected Rockwell H2RG detectors and the other nearinfrared instruments, including the NIRSpec and Fine Guidance Sensors, selected H2RG detectors shortly thereafter.

The mid-infrared instrument, MIRI, has selected arsenic-doped silicon (Si:As) detectors because Si:As meets sensitivity and other requirements. MIRI's detectors operate at temperatures $\mathrm{T} \sim 7 \mathrm{~K}$, which is much colder than the $\mathrm{T}>\sim 34 \mathrm{~K}$ temperatures that the near-infrared detectors require. These cooling requirements will be met using a solid hydrogen cryostat.

\section{MICROSHUTTERS}

Multi-object spectroscopy using JWST's Near-Infrared Spectrometer represents another quantum leap in space-based astronomy. To characterize the nature of the early universe, JWST will have to take spectral data of many different targets simultaneously. On the ground, this is relatively easy. From camera images, astronomers simply choose the objects they wish to target in their spectral studies and they create an aperture plate that allows light only from those targets to enter the spectrometer. The technique is like punching holes in a piece of cardboard.

In a multi-object spectrometer, many objects can be simultaneously placed on the slits or apertures located at the entrance field of the instrument. The Micro shutter Array (MSA) is a rectangular array of microscopic magnetically controlled, transmissive shutters, which can be opened and closed under random access control, allowing the production of any required input slit pattern.

The enabling technology for this aperture control comes from a new area of engineering called Micro Electrical Mechanical Systems (MEMS). Techniques originally developed for fabrication of integrated electronic circuits have been applied to fabrication of microscopic machines. In the MEMS approach, the punch plates used by ground-based astronomers are replaced by the array of microscopic shutters that are actuated magnetically and selected under computer control to select targets in any field on the sky.

The Goddard Space Flight Center currently is developing NASA's first-ever MEMS device that instrument makers will install in the European-built Near-Infrared Spectrometer. Featuring 268,800 microscopic shutters aligned on a silicon grid - each no larger than a dust mite - the large-format device will perform like the aperture plate used on ground-based spectrometers.

The picture below shows the set of micro shutters, each is approximately 100 millionth of a meter across, about the width of a human hair. 

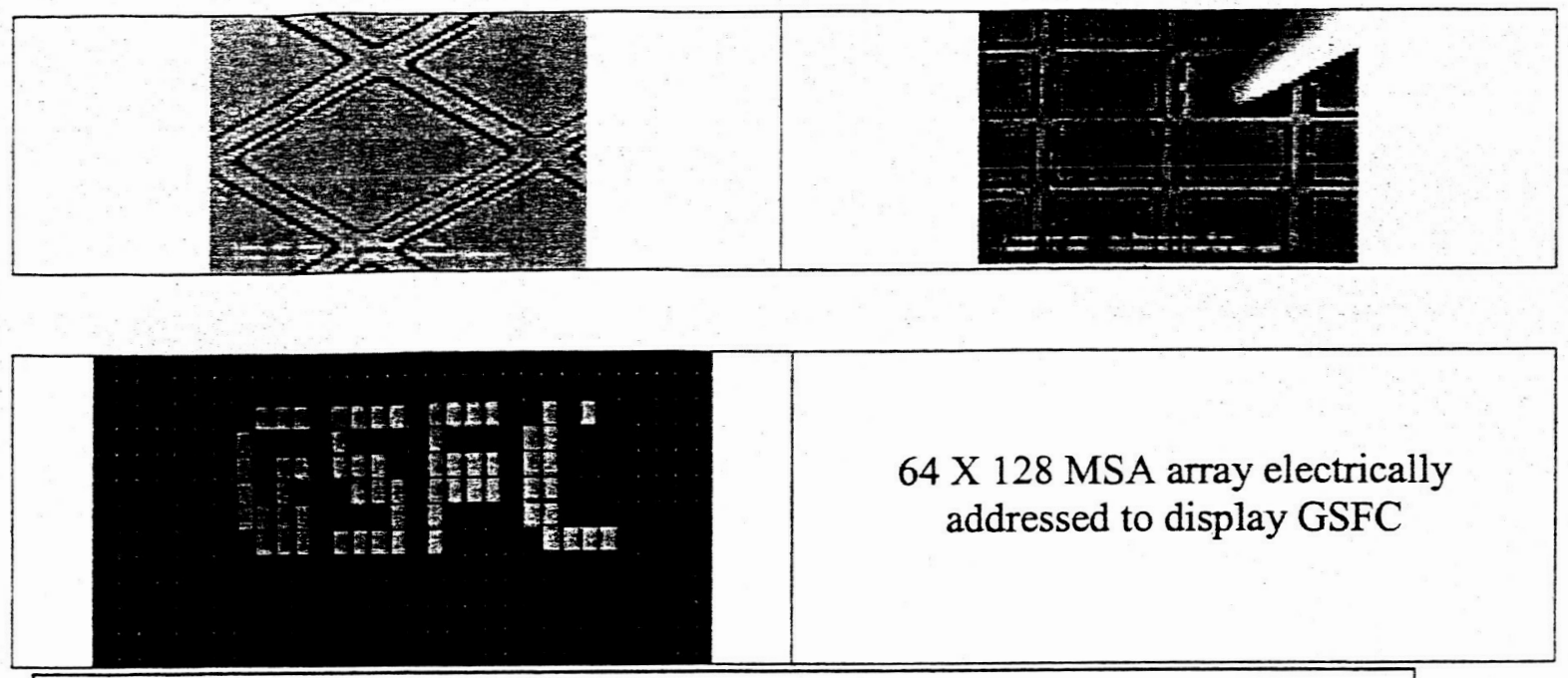

Above: Electron micrograph of a prototype JWST micro-shutter array. Light shields that mask gaps around the periphery of each shutter removed for clarity.

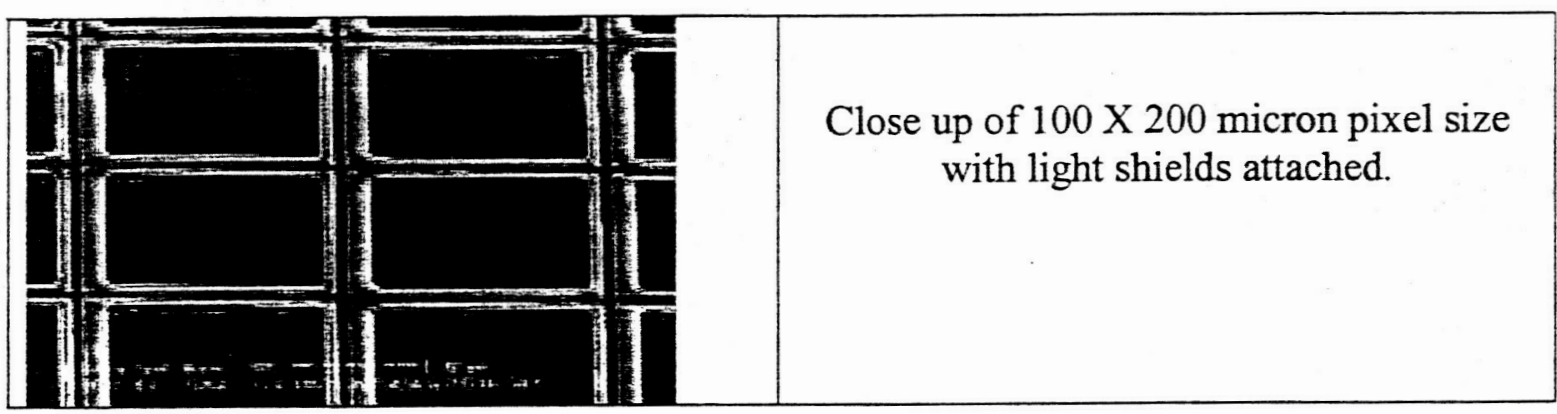

The MSA assembly consists of the following main elements, which can actuate and select the more than 250,000 individual shutters.

\section{PARDIGM-SHIFTING TECHNOLOGY INSERTION}

Through a combination of innovative systems engineering and the infusion of breakthrough technologies, like its active optics and ultra-low noise detector arrays, NASA is succeeding at containing JWST costs while meeting the scientific objectives. Competition is key to NASA's success. The guiding philosophy borne out by experience - was to keep the innovation of competition alive in the pre-development study timeframe for as long as possible. This approach engendered maximum co-investment by industry and ensured the involvement of the best engineering and scientific teams.

\section{OBSERVATORY SPACECRAFT BUS}

The spacecraft (Figure 20) provides the housekeeping function of the Observatory. It has a 471 Gigabits solid state recorder that records all science data as well as engineering data that is collected in between and during the daily contacts with the ground station. Two star trackers (an additional one for redundancy) are used to point the Observatory toward the science target prior to guide star acquisition, and they provide roll stability about the telescope line of sight (V1 axis.) Six reaction wheels ( 2 are redundant) are mounted on isolators near the center of gravity of the bus to reduce disturbances to the Observatory. These reaction wheels offload the fine steering control (operation from a $16 \mathrm{~Hz}$ update from the FGS) to maintain the fine steering mirror near its central position to limit differential distortion induced blurring onto the target star. Two fixed solar arrays canted toward the sun when the Observatory is pointed in the middle of its FOR provide power. The downlink operates at $\mathrm{Ka}$ band and has a selectable rate of 7, 14 or $28 \mathrm{Mbps}$. A pair of 
omni directional antennas (at S-band) provide near hemispherical coverage for emergency communications.

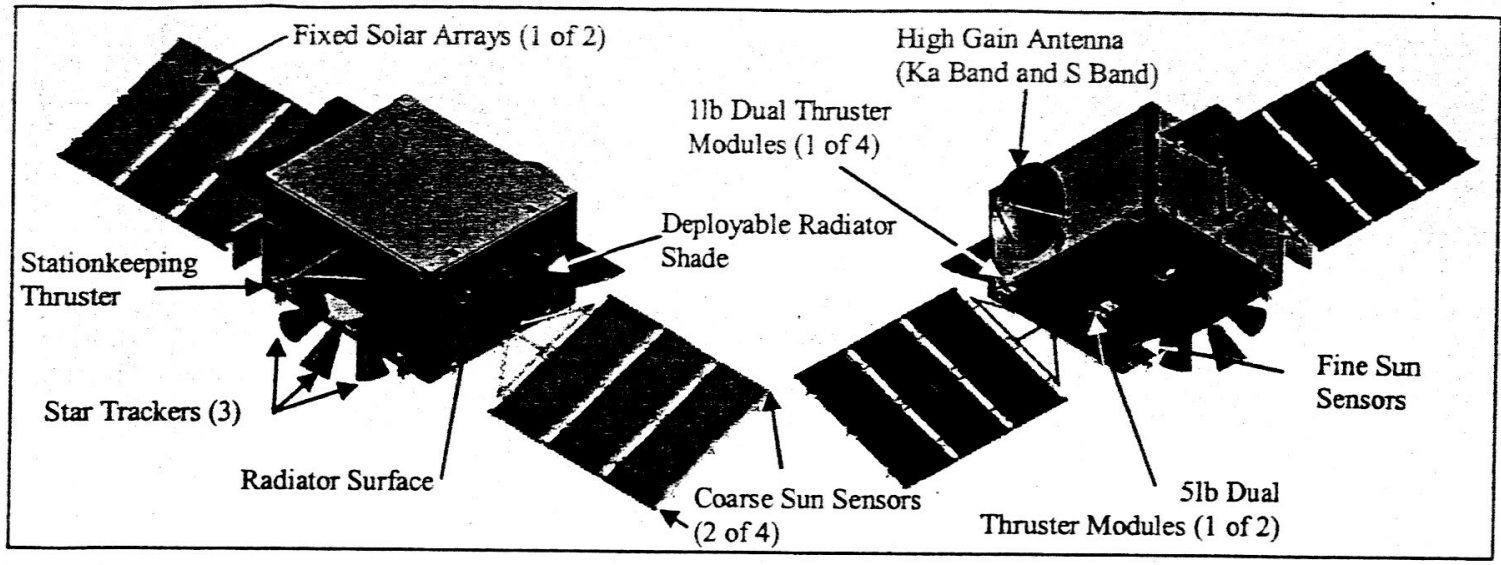

JWST Spacecraft Bus External Configuration

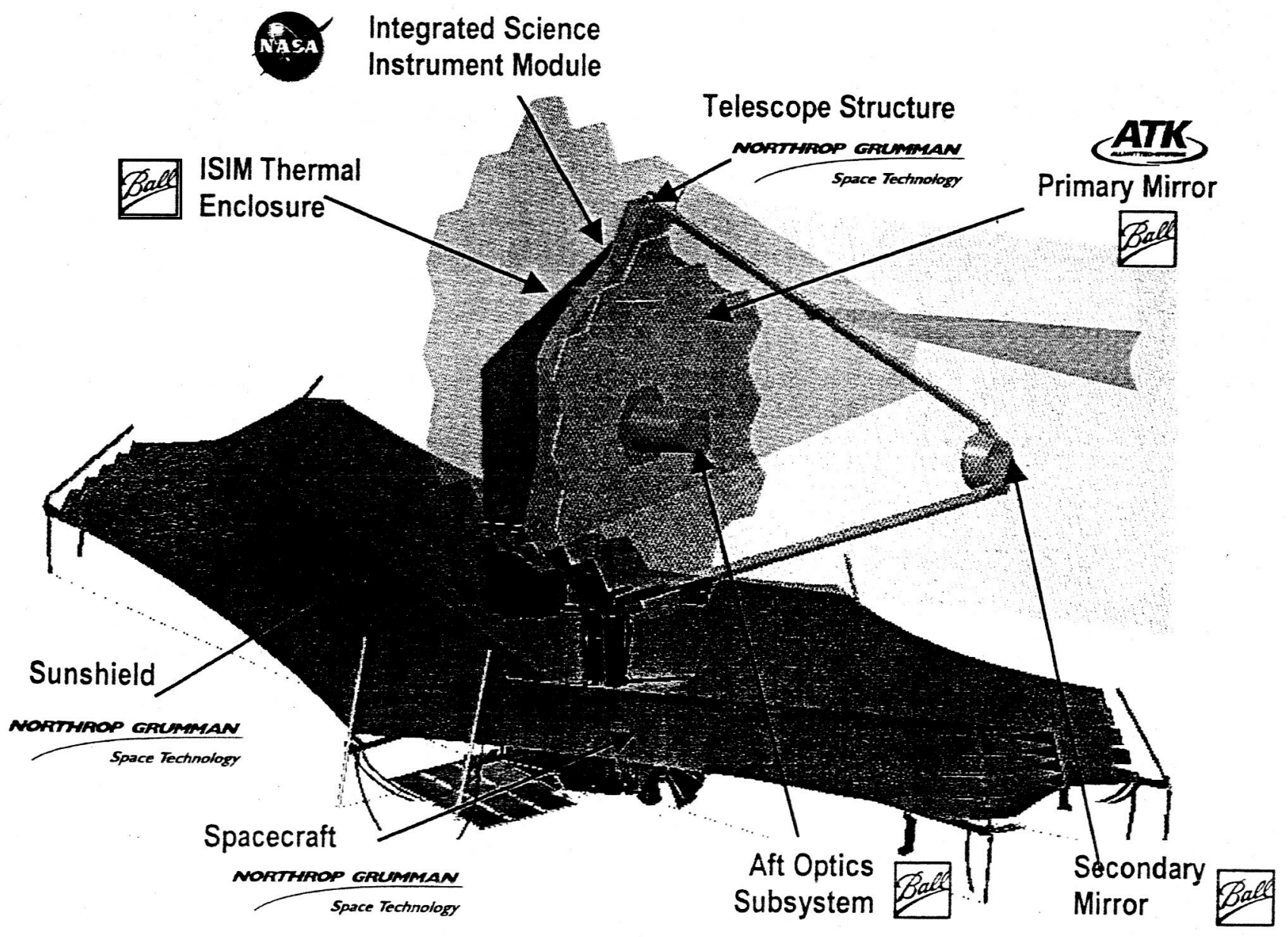




\section{PARTNERSHIPS and TEAM MEMBERS}

The table below shows the various JWST team members and their roles and responsibilities.

\begin{tabular}{|c|c|}
\hline GSFC & $\begin{array}{l}\text { Overall project management } \\
\text { - Overall systems engineering } \\
\text { - } \quad \text { Leadership of Science Working Group (SWG) } \\
\text { - } \quad \text { ISIM engineering, manufacturing and integration and test } \\
\text { Specific Engineering Directorate responsibilities; ISIM Command and Data Handling } \\
\text { (IC\&DH) with software, ISIM Structure, Micro Shutter Assembly (MSA) and } \\
\text { electronics with software and NIRSpec detectors, detector electronics, software } \\
\text { and harness }\end{array}$ \\
\hline NGST & $\begin{array}{l}\text { - Observatory systems engineering } \\
\text { - Observatory (OTE, spacecraft and ISIM) integration and test } \\
\text { - OTE, spacecraft bus and sunshield design, manufacturing, integration and test } \\
\text { - } \quad \text { Launch site processing, observatory launch and commissioning }\end{array}$ \\
\hline STScI & $\begin{array}{ll}- & \text { Ground systems development } \\
- & \text { Flight and science operations } \\
\text { - } & \text { Optics and instrument support } \\
\end{array}$ \\
\hline
\end{tabular}

\begin{tabular}{|c|c|c|}
\hline 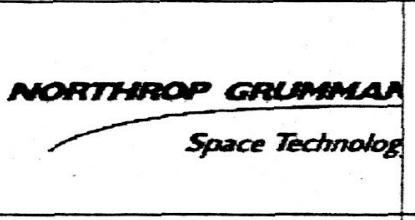 & $\begin{array}{l}\text { JWST Prime } \\
\text { Contractor }\end{array}$ & $\begin{array}{l}\text { - } \quad \text { Observatory performance and programmatics } \\
\text { - Systems engineering and interfaces } \\
\text { - } \quad \text { Obacecraft, Sunshield, and deployables } \\
\text { - Support ground segment and operations } \\
\end{array}$ \\
\hline & $\begin{array}{l}\text { Optical System } \\
\text { Development }\end{array}$ & $\begin{array}{l}\text { - Optical Telescope Element (OTE) optical } \\
\text { design and optics } \\
\text { - Beryllium mirror segment development and } \\
\text { cryogenic testing } \\
\text { - Wavefront Sensing \& Control (WFS\&C) } \\
\text { design and algorithms } \\
\text { - OTE and Observatory AI\&T support }\end{array}$ \\
\hline & $\begin{array}{l}\text { Telesopelntegration } \\
\text { and Test }\end{array}$ & $\begin{array}{ll}\text { - } & \text { OTE ground AI\&T } \\
\text { - } & \text { Plum Brook test configuration and interfaces }\end{array}$ \\
\hline & Telescope Structure & $\begin{array}{l}\text { Telescope backplane and Secondary Mirror } \\
\text { (SM) support structure design and build }\end{array}$ \\
\hline
\end{tabular}

\begin{tabular}{|c|c|}
\hline ESA/European Consortium & $\begin{array}{l}\text { - NIRSpec Instrument } \\
\text { - MIRI OBA(Optical Bench Assembly) and instrument integration \& test } \\
\text { - Ariane } 5 \text { launch vehicle (TBR) }\end{array}$ \\
\hline CSA & - FGS with tunable filter modules \\
\hline Uof $A z$ & - NIRCam instrument (LM ATC is prime) \\
\hline JPL & $\begin{array}{l}\text { - MIRI management and systems engineering team lead, flight software, } \\
\text { FPA/FPE and dewar } \\
\text { - Wave Front Sensing and Control (WFS\&C) technology development } \\
\text { and oversight }\end{array}$ \\
\hline MSFC & $\begin{array}{l}\text { - Mirror technology development and testing } \\
\text { - Environmental analysis }\end{array}$ \\
\hline AMES & - Detector technology development \\
\hline
\end{tabular}


International partnering is an important way for NASA to keep down costs for this order-of-magnitude increase in scientific capability over that of Hubble. The European Space Agency (ESA) is contributing about $\$ 200$ million (U.S.) for a 15 percent observing share and the Canadian Space Agency (CSA) will contribute more than $\$ 50$ million for its roughly 5 percent share. Truly an international endeavor, the JWST science teams also include private-sector partners from Canada and Europe.

In addition to its partnerships with Europe and Canada, NASA has relied on the expertise of its field centers, including the Goddard Space Flight Center, Ames Research Center, the Jet Propulsion Laboratory and the Marshall Space Flight Center, and several of the Department of Energy's national laboratories. The Space Telescope Science Institute, the same organization that now operates Hubble, will operate JWST's Science and Operations Center. Universities and a variety of industry groups scattered across the country are also involved in one form or another with the JWST program.

The Department of Defense (DoD) contributed to the technology development effort. It helped to fund the joint advanced mirror technology-development (AMSD) program that led to the development of semi-rigid beryllium mirrors chosen for JWST.

\section{MISSION OPERATIONS}

Mission operations are conducted through the JWST ground system, which includes the Deep Space Network (DSN), NASA Integrated Services Network (NISN), Goddard Space Flight Center's Flight Dynamics Facility (FDF) and the Science and Operations Center (S\&OC). The S\&OC is responsible for operating the Observatory, and is being developed and staffed by the Space Telescope Science Institute. The S\&OC enables the planning and execution of scientific investigations. The remainder of the ground segment enables tracking and communications, housekeeping, and maintenance of the Observatory.

The JWST architecture has driven the design of the ground system architecture. JWST is designed to operate autonomously on orbit at the L2 for extended periods of time through the incorporation of eventdriven, rather than time-driven, activity management. This will permit 8-hour per day, 5-day per week staffing of the S\&OC for most operations. 


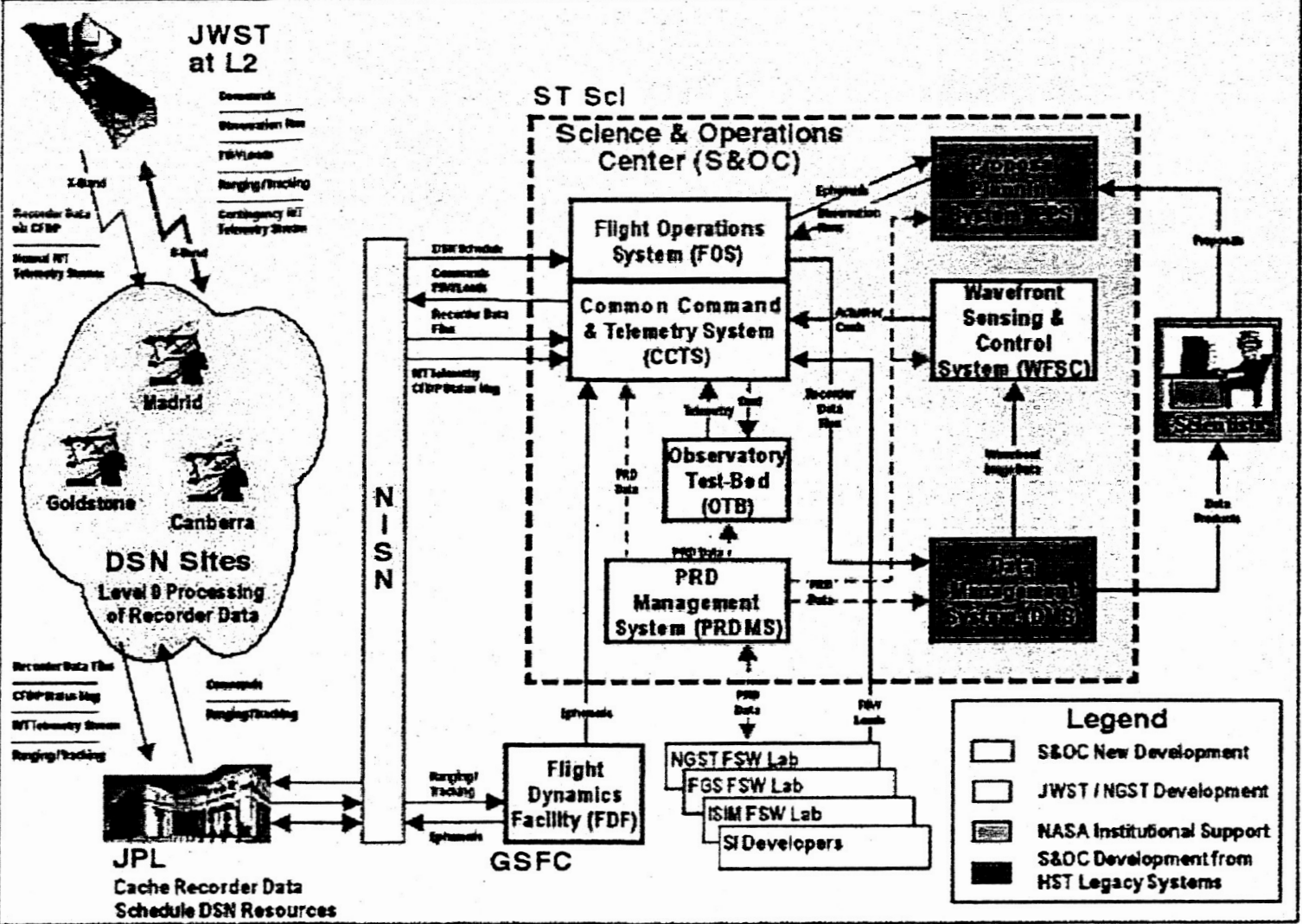

\section{CONCLUSION}

JWST is vital, affordable, and scientifically capable of remaining as the National Academy of Science's top investment priority for NASA Space Astronomy this decade. It is a model of international cooperation and collaboration and it continues to thrive under a philosophy that demands clear, centralized management and strong systems engineering. As of today, the team has proven all major technologies, including the viability of lightweight active optics and image-based wavefront sensing and control. Now, in partnership with Northrop Grumman as the prime contractor, the JWST project officially moves to the next phase of its development and a step closer to launching a new era of astronomical discovery. In the end, JWST will touch and inspire the lives of thousands of scientists and engineers from across the United States, Canada and Europe, to say nothing of the astronomers worldwide, whose discoveries using JWST may well change the way we see ourselves and our place in the universe.

\section{ACKNOWLEDGMENTS}

The authors thank the many men and women working on JWST, particularly NASA's industry, and international partners both here and abroad.

\section{REFERENCES}

1. A. Dressler et al., HST and Beyond; Explorations and the Search for Astronomical Origins: A Vision for Ultraviolet-Optical-Infrared Space Astronomy, AURA, 1996.

2. H.S. Stockman, Next Generation Space Telescope: Visiting a Time When Galaxies Were Young, AURA, 1997.

3. National Academy of Science decadal report (that says that JWST is NASA's most important space science mission. 